\title{
Synergistic interaction of 5-aminolevulinic acid-based photodynamic therapy with simultaneous hyperthermia in an osteosarcoma tumor model
}

\author{
SIGEAKI YANASE, JOUJI NOMURA, YOSHIHIKO MATSUMURA, \\ TAKAYUKI NAGATA, TASUKU FUJII and TOSHIRO TAGAWA \\ Department of Oral and Maxillofacial Surgery, Faculty of Medicine, \\ Mie University, 2-174 Edobashi, Tsu, Mie 514-8507, Japan
}

Received January 27, 2006; Accepted March 22, 2006

\begin{abstract}
In this study, the effectiveness and mechanism of combination therapy of 5-aminolevulinic acid-based photodynamic therapy (ALA-PDT) with simultaneous hyperthermia (HT) on human-derived osteosarcoma in vivo were examined. A tumor model was prepared by subcutaneously implanting human osteosarcoma into nude mice and local injection of ALA was selected as the administration route. This study demonstrated that both ALA-PDT and the combination of ALA-PDT with HT exhibited significant inhibitory effects on tumor growth. In the group with high total energy, the growth inhibition rates of tumor were $52.3 \%$ in ALA-PDT, and $27.3 \%$ in ALA-PDT with simultaneous HT (PDT+HT), and the synergetic index was 1.76 , demonstrating that the inhibitory effect on tumor growth in ALA-PDT was significantly increased by simultaneous use of HT. In the histological findings, after ALA-PDT, necrosis was observed in the area from the surface to a depth of $2 \mathrm{~mm}$, and only a slight effect was confirmed in deeper layers. On the other hand, after PDT $+\mathrm{HT}$, necrosis was observed in layers even deeper than $2 \mathrm{~mm}$ below the surface. Furthermore, based on the immunohistochemical findings of the expression of carbonic anhydrase IX, while weak expression of CAIX was observed after ALA-PDT in an area relatively close to the surface, a positive area extended to the deeper layers after PDT+HT compared with ALA-PDT. In conclusion, PDT + HT demonstrated a significant inhibitory effect on tumor growth of human-derived osteosarcoma, and a synergistic interaction of simultaneous HT. This suggests the possibility that ALA-PDT is useful, not only for the treatment of superficial tumors but also for deep-seated mesenchymal tumors, such as osteosarcoma. Furthermore, the mechanism of the synergistic interaction suggested that ALA-PDT was
\end{abstract}

Correspondence to: Dr Shigeaki Yanase, Department of Oral and Maxillofacial Surgery, Faculty of Medicine, Mie University, 2-174 Edobashi, Tsu, Mie 514-8507, Japan

E-mail: shigeaki@clin.medic.mie-u.ac.jp

Key words: 5-aminolevulinic acid, photodynamic therapy, hyperthermia, osteosarcoma, simultaneous use effective in the deep area of tumors due to the increase of blood flow by mild hyperthermia.

\section{Introduction}

In photodynamic therapy (PDT), a light-activated agent (a photosensitizer: PS) is accumulated in the target tissue, and is then activated by irradiation at an appropriate wavelength to induce production of reactive oxygen species (mainly singlet oxygen) resulting in cytotoxicity and cell damage. This therapy has been used for the treatment of various malignant diseases (1).

5-Aminolevulinic acid (ALA) itself is not a PS, but is a precursor of endogenous porphyrins in the heme biosynthesis pathway, that activates production of a PS, protoporphyrin IX (PPIX), in mitochondria by bypassing the negative feedback control of heme (2). PPIX in the ground state absorbs energy from light at a wavelength of $\sim 630 \mathrm{~nm}$, and the energy is transferred from the excited state PPIX to molecular oxygen. This interaction generates cytotoxic reactive oxygen species, and mainly generates singlet oxygen, which can then kill cells. Since PPIX mainly accumulates in mitochondria, these organelles may be the specific target of the cytotoxic photochemical reaction in PDT with ALA (ALA-PDT) (3). While direct cell damage by ALA-PDT has been observed in vitro, it has been demonstrated that ALA-PDT in vivo did not cause severe damage, such as reduction in blood flow at the tumor site, resulting in less damage on vascular endothelial cells; although the effect of PDT on tumor blood vessels is generally considered to be an important factor of antitumor treatment (4).

On the other hand, it is well known that hyperthermia (HT) causes cell death. At the cellular level, HT at $42^{\circ} \mathrm{C}$ or higher causes direct damage to cells, damage to DNA, serious destruction of the plasma membrane, inhibition of protein synthesis in conjunction with energy metabolism, and mitochondrial damage (5-8). However, the mechanism of cell damage in animal tumor models is considered to be different from that at the cellular level, as HT affects tumor blood vessels. HT at $42.5^{\circ} \mathrm{C}$ or higher for $1 \mathrm{~h}$ causes damage to blood vessels and a decrease in blood flow at the tumor site, inducing hypoxia, low $\mathrm{pH}$ and hypoalimentation in the tumor (9). Furthermore, mild HT at $38-41^{\circ} \mathrm{C}$ may cause an increase in blood flow (8). 
In recent years, local application of ALA-PDT has been used successfully to treat various superficial diseases. Since ALA is administered locally, and has low permeability, production of protoporphyrin IX (PPIX) is limited to the surface and the treatment is considered to be ineffective for deep-seated nodular tumor (10). However, since ALA does not exhibit photosensitivity and causes no serious adverse effects, such as those observed in use of Photofrin (3), ALA can be used repeatedly at the same site, and is therefore considered to be useful in clinical practice $(11,12)$. If combination therapy of ALA-PDT and HT is effective, not only for superficial diseases but for deep-seated diseases, broader treatment options will be available for neoplastic lesions, including malignant tumors. However, there have been few in vivo studies of combination therapy for deep-seated solid tumors $(13,14)$, and no studies using human-derived osteosarcoma have been reported.

We remodeled a linearly polarized near infrared hyperthermic irradiator [Super Lizer ${ }^{\circledR}$ (SL)], which has been widely used in the infrared therapy for pain, to enable simultaneous irradiation of red light used in ALA-PDT and near infrared light resulting in HT. Using the modified SL, we examined the effect of combination therapy of ALA-PDT and HT on human osteosarcoma in vitro (11). As a result, it was confirmed that the effect of ALA-PDT to kill cells would be significantly enhanced with simultaneous use of HT, and that one of the mechanisms of the synergistic interactions would be increase of mitochondrial damage.

In this study, a tumor model was prepared by subcutaneously implanting human osteosarcoma into nude mice to examine the effect and mechanism of action of simultaneous treatment with ALA-PDT and HT.

\section{Materials and methods}

Animals and tumors. Female BALB/cAjcl-nu mice (Japan clea, Osaka, Japan; 6-weeks old, weight range 19-22 g at the time of tumor implantation) were used for all experiments. A transplantable human osteosarcoma tumor model from the mandible of nude mice (HOSMN-2) was established and retained by our department (12). Tumors were propagated by the subcutaneous implantation of tumor pieces from donor mice. In the current experiment, tumors that exhibited a surface diameter of 7-9 $\mathrm{mm}$ within 10-14 days after implantation were used.

Chemicals. 5-Aminolevulinic acid hexylester hydrochloride (hALA) (Cosmo Bio Co., Tokyo, Japan) was dissolved in phosphate-buffered saline (PBS) at a final concentration of $250 \mathrm{mg} / \mathrm{ml}$. This solution was slowly injected subcutaneously into the area close to the tumor so that each mouse received a dose of $250 \mathrm{mg} / \mathrm{kg}$ hALA (i.e., a final volume of $\sim 0.2 \mathrm{ml}$ ).

Light source. The specifications of the Super Lizer (model HA-550; Tokyo Iken, Co., Ltd. Tokyo, Japan) (SL) are: wavelength, 600-1600 nm; power output, $1800 \mathrm{~mW}$; weight, $24 \mathrm{~kg}$; dimensions, 370x510x990 mm. The attachment of two newly prepared filters (consisting of a band pass filter through which rays with wavelengths of 580-740 nm and $940 \mathrm{~nm}$ or longer can penetrate, and a short pass filter through which rays with a wavelength of $750 \mathrm{~nm}$ or shorter can penetrate) allows the irradiator to generate two types of rays. Hence, one type has a wavelength range from 580 to $740 \mathrm{~nm}$, which includes the appropriate wavelength for PPIX, while the other has a wavelength range from 940 to $1600 \mathrm{~nm}$, which includes near infrared rays that serve to increase the temperature. In ALA-PDT, rays ranging from 580 to $740 \mathrm{~nm}$ can be generated by utilization of a combination of the band pass filter and the short pass filter, and the maximum output is $110 \mathrm{~mW}$ (Fig. 1a). In ALA-PDT combined with simultaneous HT, rays are generated by attachment of the band pass filter only, and wavelengths ranging from 580 to $740 \mathrm{~nm}$ and from 940 to $1600 \mathrm{~nm}$ are simultaneously utilized. The maximum output in this case is $710 \mathrm{~mW}$ (Fig. 1b). In the current study, 580-740 nm rays were used for ALA-PDT, and it was confirmed that these rays do not raise the temperature in the tumor.

Hyperthermic conditions. The temperature in the tumor was monitored by a digital microprobe thermometer that was inserted into the tumor from the side and set at depths of 2, 4 and $6 \mathrm{~mm}$ under the surface. The time-course for the change in the temperature was recorded every $30 \mathrm{sec}$ during irradiation. The room temperature was maintained at approximately $20^{\circ} \mathrm{C}$.

Intratumoral accumulation of protoporphyrin IX. The accumulation of protoporphyrin IX (PPIX) in the tumor was investigated by measuring the fluorescence of tumor extract at various time points after injection of hALA. Tumor bearing mice injected with hALA were maintained under light hind until the tumor excision. The tumor was excised at set time points $1,3,5$, and $7 \mathrm{~h}$ after injection). Tumor samples were rinsed with $0.9 \% \mathrm{NaCl}$ and homogenized in $300 \mu \mathrm{l} \mathrm{PBS}$. Aliquots of tumor homogenates (200 $\mu \mathrm{l})$ were added to $200 \mu \mathrm{l}$ of solvent containing $1 \%$ sodium dodecyl sulfate (SDS) in $1 \mathrm{~N}$ perchloric acid $\left(\mathrm{HClO}_{4}\right)$ and methanol $\left(\mathrm{CH}_{3} \mathrm{OH}\right)(1: 1 \mathrm{v} / \mathrm{v})$, were homogenized again, and then kept frozen overnight at $-20^{\circ} \mathrm{C}$. Frozen mixtures were thawed at room temperature, sonicated for $1 \mathrm{~min}$, and then centrifuged at $15000 \mathrm{x} \mathrm{g}$ for $30 \mathrm{~min}$. A $300-\mu 1$ aliquot of each supernatant was transferred to 96-well plates for fluorescence detection (Sumitomo Bakelite Co., Ltd., Tokyo, Japan), and the fluorescence intensity was measured by fluorescence spectrophotometry. The fluorescence excitation wavelength was $380 \mathrm{~nm}$ and emission wavelength was $612 \mathrm{~nm}$. To normalize PPIX content to protein content, PPIX was dissolved in the solvent described above. The fluorescence intensity of $0-100 \mu 1 / \mathrm{ml}$ of PPIX solutions was measured by fluorescence spectrophotometry to generate a PPIX standard curve. The protein concentration was measured using a BCA protein assay kit (Pierce Rockford, IL, USA). Aliquots $(20 \mu \mathrm{l})$ of the tumor homogenates were added to $20 \mu 1$ of buffer containing $1 \%$ Triton $\mathrm{X}-100,0.1 \%$ SDS, $0.5 \%$ sodium deoxycholate, $100 \mathrm{mM} \mathrm{NaCl}, 1 \mathrm{mM}$ phenylmethylsulfonyl fluoride, $20 \mu \mathrm{g} / \mathrm{ml}$ aprotinin, and $20 \mu \mathrm{g} / \mathrm{ml}$ leupeptin and lysed at $4^{\circ} \mathrm{C}$ for $30 \mathrm{~min}$. The mixtures were centrifuged at $16000 \mathrm{x} \mathrm{g}$ for $30 \mathrm{~min}$, and the supernatants were transferred to the tube, and protein concentrations were determined according to the manufacturer's instructions. Following determination of the PPIX content and protein concentrations, the values of PPIX content per unit protein $(1 \mathrm{mg})$ in the tumor was calculated. 
(a) For ALA-PDT
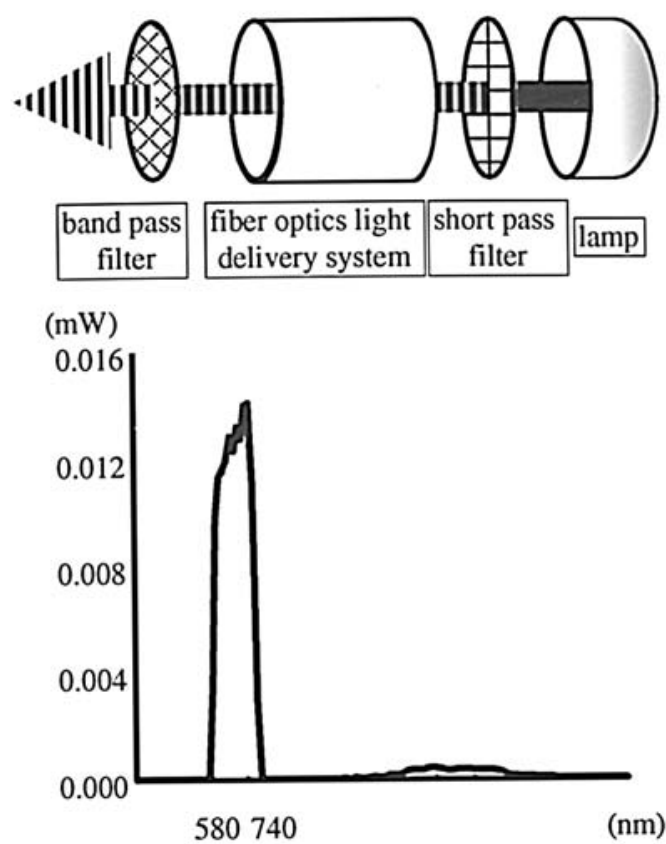

(b) For ALA-PDT combined with simultaneous HT
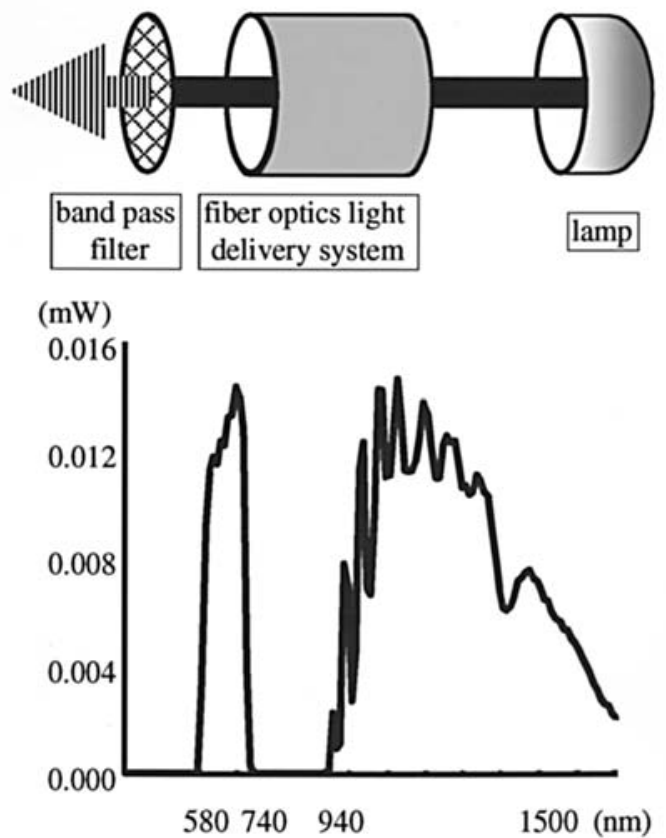

Figure 1. Properties of the output wavelength. (a) For ALA-PDT: wavelength, 580-740 nm (including the appropriate wavelength for PPIX excitation); power output, $110 \mathrm{~mW}$. (b) For ALA-PDT combined with simultaneous HT: wavelength, 580-740 nm and 940-1600 nm (including the appropriate wavelength for PPIX excitation and increasing temperature); power output, $710 \mathrm{~mW}$.

Treatments. Once the surface diameter of the tumor reached 7-9 $\mathrm{mm}$, animals were anesthetized with ketamine and randomly allocated to the various experimental groups $(n=3)$. The untreated control group received no treatment. Treatment groups were divided into two large groups, the L-group, which was treated with low total energy (irradiation time: $24 \mathrm{~min}$ ), and the H-group, which was treated with high total energy (irradiation time: $40 \mathrm{~min}$ ). Each group included a hyperthermia alone group (HT), an ALA-PDT group (PDT) and an ALAPDT combined with simultaneous hyperthermia group (PDT+HT). Thus, a total of six treatment groups were used. In other words, the L-group includes HT-L (irradiated for hyperthermia alone), PDT-L (after hALA injection and light exposure for $3 \mathrm{~h}$, the animals underwent PDT) and PDT+HT-L (PDT occurred simultaneously with hyperthermia), and the H-group includes HT-L, PDT-L and PDT+HT-L similar to the L-group (Table I).

Evaluation of the tumor response. Tumor volume was calculated as $\mathrm{V}=(\mathrm{a} \times \mathrm{b} \times \mathrm{b}) / 2$ where $\mathrm{a}$ is the major axis and $b$ is the minor axis. The antitumor effect of the treatment was determined as the growth rate of the tumor (GR), Ve/Vc (the tumor volume of the experimental group, respectively, at 12 days after treatment and compared with that of untreated controls). The growth inhibition rate (GIR), was calculated as Gre/GRc, where GRe and GRc are the GIR of the experimental-group and that of untreated control. To determine the combined effect of HT and PDT, a synergistic index (SI), GIR in the HT alone/GIR in the PDT alone was calculated according to Valeriote's criteria (15).

Histological findings. Histological alterations were examined at $24 \mathrm{~h}$ after each treatment. Specimens were fixed with $10 \%$
Table I. Treatment schema.

\begin{tabular}{lccc}
\hline Each group & $\begin{array}{c}\text { Fluence rate } \\
\left(\mathrm{mW} / \mathrm{cm}^{2}\right)\end{array}$ & $\begin{array}{c}\text { Total dose } \\
\left(\mathrm{J} / \mathrm{cm}^{2}\right)\end{array}$ & $\begin{array}{c}\text { Irradiation time } \\
(\mathrm{min})\end{array}$
\end{tabular}

Untreated

Low energy

dose group

$\begin{array}{lcrl}\text { HT-L } & 310 & 440 & 24 \\ \text { PDT-L } & 35.4 & 50 & 24 \\ \text { PDT+HT-L } & 310 & 440 & 24\end{array}$

High energy

dose group

\begin{tabular}{lcrr} 
HT-H & 310 & 740 & 40 \\
PDT-H & 35.4 & 85 & 40 \\
PDT+HT-H & 310 & 740 & 40 \\
\hline
\end{tabular}

formalin and embedded in paraffin. Four micron thick slides were prepared and stained with hematoxylin and eosin.

Immunohistochemical analysis of carbonic anhydrase IX expression. Immunostaining of paraffin sections was performed after dewaxing and rehydrating the $4-\mu \mathrm{m}$ sections. For carbonic anhydrase IX (CAIX) detection, antibodies specific for CA IX (rabbit polyclonal antibody; Santa Cruz Biotechnology, CA, USA) diluted 1:500 in PBS as a primary antibody and an automated immunostainer NexES IHC staining module (Ventana Medical Systems, Inc., Tuson, AZ, USA) was used. 


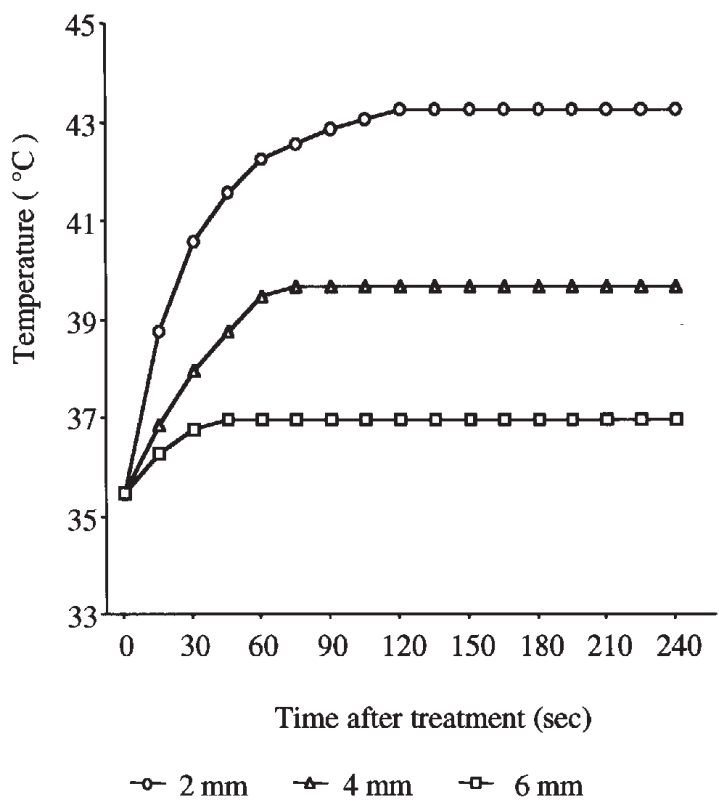

Figure 2. Temperature at depths of 2, 4, $6 \mathrm{~mm}$ inside the tumor in nude mice without hALA injection during 580-740 and 940-1600 $\mathrm{nm}$ irradiation at a fluency rate of $310 \mathrm{~mW} / \mathrm{cm}^{2}$.

Statistical analysis. The entire experiment was performed in triplicate and assessed statistically using the Student's t-test. Significance was defined as a calculated $\mathrm{p}<0.05$.

\section{Results}

Hyperthermic conditions. During irradiation in HT or PDT+HT, the temperature of the tumor reached $43.3^{\circ} \mathrm{C} \sim 2$ min after initiation of the irradiation at a depth of $2 \mathrm{~mm}$ from the tumor surface; $39.7^{\circ} \mathrm{C}$ after $\sim 1.5 \mathrm{~min}$ at a depth of $4 \mathrm{~mm}$; and $37^{\circ} \mathrm{C}$ after $\sim 45 \mathrm{sec}$ at a depth of $6 \mathrm{~mm}$. Individual temperatures were kept stable during the irradiation (Fig. 2).

Intratumoral accumulation of protoporphyrin IX. Intratumoral accumulation of PPIX was $44.31 \pm 45.17 \mathrm{ng} / \mathrm{mg}$ protein before application of hALA, and increased significantly after hALA application. After reaching a maximum of $908.72 \pm 314.6 \mathrm{ng} / \mathrm{mg}$ protein $(\mathrm{p}=0.039), 3 \mathrm{~h}$ after the application, the accumulation was reduced to $313.15 \pm 242.75 \mathrm{ng} / \mathrm{mg}$ protein $7 \mathrm{~h}$ after the application (Fig. 3).

Tumor response. In the investigation of the effect on increase in tumor volume after individual treatments, the GR of the untreated group was $7.58 \pm 0.38$ on day 12 , and there was no significant difference compared with the GR of the HT-H group (also not significantly different from the hALA alone group; data not shown). The HT-L group (GR: 6.56 \pm 0.12 ), PDT-L group (GR: $5.28 \pm 0.59)$ and PDT+HT-L group (GR: 3.37 \pm 0.50 ) demonstrated significant inhibitory action on tumor growth, and significant differences were evident between the individual treatment groups. In addition, the PDT-H (GR: 3.88 \pm 0.60$)$ and PDT+HT-H group (GR: 2.07 \pm 0.08 ) also exhibited significant inhibitory action on tumor growth inhibition compared with the untreated group, and significant differences were confirmed between the individual treatment groups (Fig. 4 and Table II).

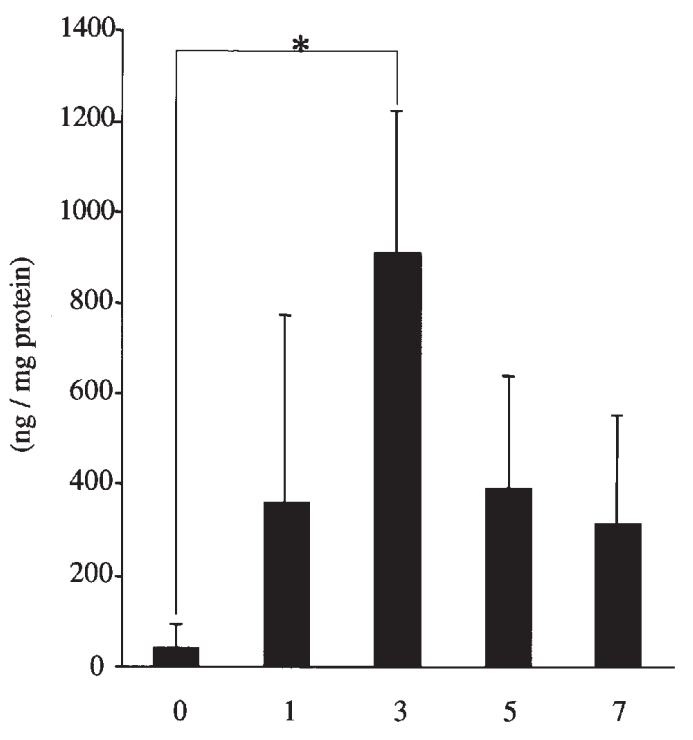

Time after application (hr)

Figure 3. Intratumoral accumulation of protoporphyrin IX. Animals were injected with hALA $(250 \mathrm{mg} / \mathrm{kg}$, dissolved in $0.2 \mathrm{ml} \mathrm{PBS})$ in the periphery of the tumor. After 3, 5, $7 \mathrm{~h}$, tumors were enucleated. PPIX was extracted from both treated and untreated tumors. Each data point represents the mean \pm SD. The experiment was performed at least three times. ${ }^{*} \mathrm{P}<0.05$.

The tumor growth inhibition rates (GIR) on day 12 were $69.6 \%$ in the PDT-L group, $49.2 \%$ in the PDT+HT-L group, $52.3 \%$ in the PDT-H group, and $27.3 \%$ in the PDT+HT-H group (Table III). The synergetic indices were 1.22 for the $\mathrm{L}$ group and 1.76 for the $\mathrm{H}$ group, and both groups demonstrated a synergetic interaction between combination therapy of ALA-PDT and hyperthermia (Table IV).

Histological findings. In the investigation of histological changes $24 \mathrm{~h}$ after treatment, tumor cells in a circular or polygonal shape with mitotic form were observed beneath the epithelium in the untreated group (Fig. 5a). In the HT-H group, bleeding was observed at a depth of $\sim 1 \mathrm{~mm}$ from the surface with a small necrotic area in the region from the surface to a depth of $2 \mathrm{~mm}, 24 \mathrm{~h}$ after the treatment (Fig. 5b). In the PDT-H group, necrosis was observed in deeper layers than in the HT-H group, $24 \mathrm{~h}$ after the treatment. However, the necrotic area was limited to the depth of $2 \mathrm{~mm}$ from the surface, and the effect observed in the layers below this was slight (Fig. 5c). The necrotic area expanded to deeper layers $24 \mathrm{~h}$ after treatment in the PDT+HT-H group compared with the HT-H group. In addition to the destruction of the epithelial layer and atrophied tumor cells of the deep layers, the necrotic area expanded to the layers below the depth of $2 \mathrm{~mm}$ from the surface in the PDT+HT-H group (Fig. 5d).

Immunohistochemical findings. In the immunohistochemical examination of the expression of carbonic anhydrase IX, a positive area was observed in the bleeding layer immediately under the epithelium and in the deeper layers $24 \mathrm{~h}$ after treatment in the HT-H group (Fig. 6b), while a positive area was confirmed only in a portion of the epithelium in the untreated group (Fig. 6a). In the PDT-H-group, a weak but larger positive 


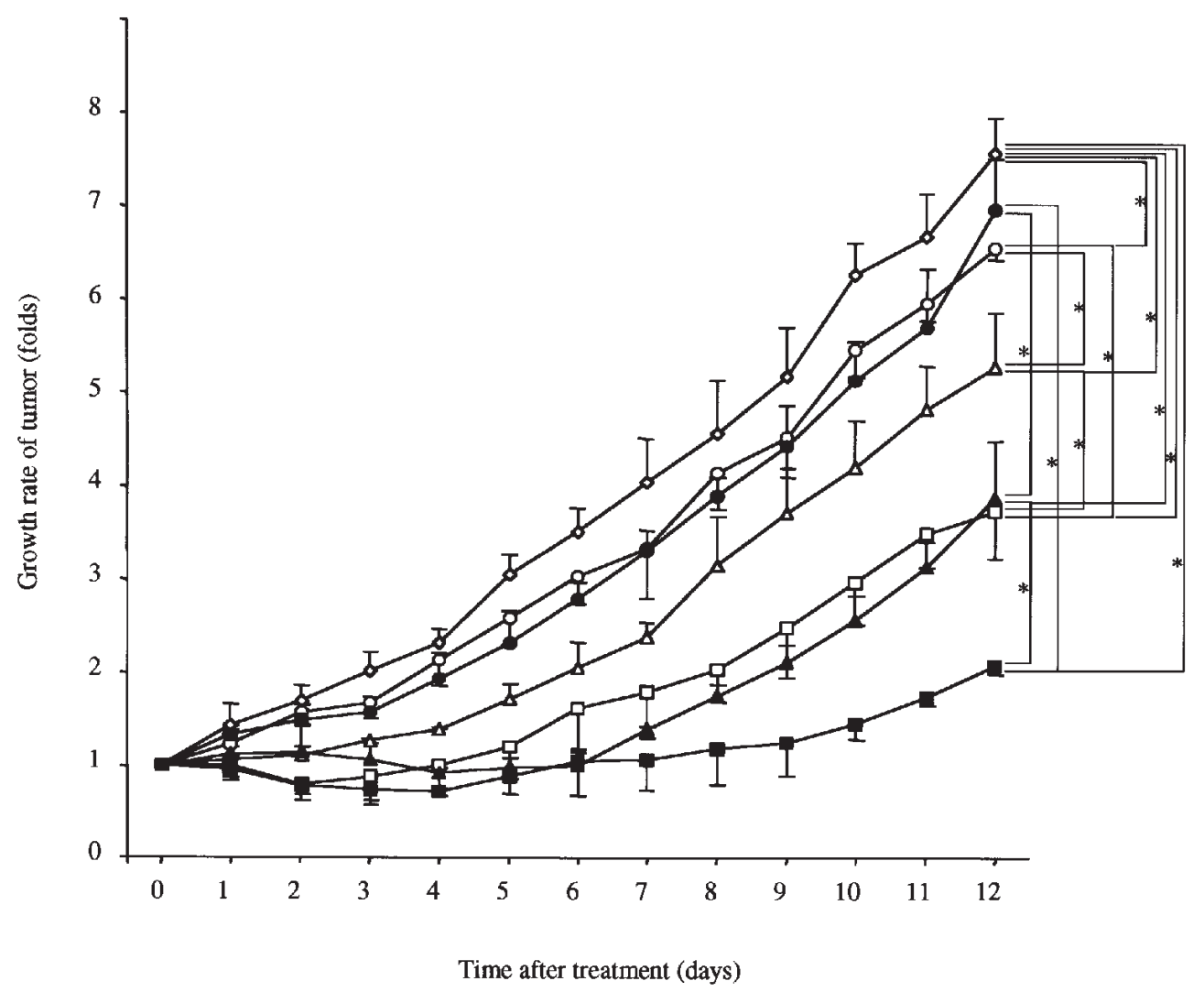

$\multimap$ untreated $\multimap-$ HT-L $\rightarrow$ PDT-L $\rightarrow$ - PDT+HT-L

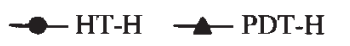

PDT+HT-H

Figure 4. Effect of treatment on the growth of tumors. Each data point represents the mean $\pm \mathrm{SD}$. The experiment was performed at least three times. ${ }^{*} \mathrm{P}<0.05$.

Table II. Growth rate of tumors (GR).

GR on day-12 (folds)

\begin{tabular}{ll}
\hline Untreated & $7.58 \pm 0.38$ \\
HT-L & $6.56 \pm 0.12^{\mathrm{a}, \mathrm{b}}$ \\
PDT-L & $5.28 \pm 0.59^{\mathrm{a}, \mathrm{b}}$ \\
PDT+HT-L & $3.73 \pm 0.50^{\mathrm{a}, \mathrm{b}}$ \\
HT-H & $6.97 \pm 0.54^{\mathrm{a}}$ \\
PDT-H & $3.88 \pm 0.60^{\mathrm{a}, \mathrm{b}}$ \\
PDT+HT-H & $2.07 \pm 0.08^{\mathrm{a}, \mathrm{b}}$ \\
\hline
\end{tabular}

Data are the growth rates of tumors and are expressed as the mean \pm $\mathrm{SD}$ of three mice. ${ }^{a}$ Difference is statistically significant $(\mathrm{p}<0.05)$.

area was confirmed in layers deeper than those observed in the HT-H group $24 \mathrm{~h}$ after treatment (Fig. 6c). In the PDT+HT-H group, decreased expression of carbonic anhydrase IX was observed at the epithelial layer compared to the PDT-H group, $24 \mathrm{~h}$ after treatment (Fig. 6d), and the strongly positive area expanded to the deeper layers of the tumor.

\section{Discussion}

In the 1960s, Liposon et al developed a hematoporphyrin derivative $(\mathrm{HpD})$ and reported the efficacy of photodynamic
Table III. Growth inhibition rate (GIR).

\begin{tabular}{lc}
\hline & GIR on day-12 $(\%)$ \\
\hline HT-L & 86.6 \\
PDT-L & 69.6 \\
PDT+HT-L & 49.2 \\
HT-H & 91.9 \\
PDT-H & 52.3 \\
PDT+HT-H & 27.3 \\
\hline
\end{tabular}

Table IV. Synergistic index (SI).

SI on day-12

\begin{tabular}{ll}
\hline PDT+HT-L & 1.22 \\
PDT+HT-H & 1.76 \\
\hline
\end{tabular}

therapy (PDT) using HpD for the treatment of cancer. Since then, several photosensitizers have been developed (12), and recent reports have shown that PDT is an excellent therapy for various diseases, including malignant tumors of the skin, prostate, uterus, lungs and stomach (16). At present, further 


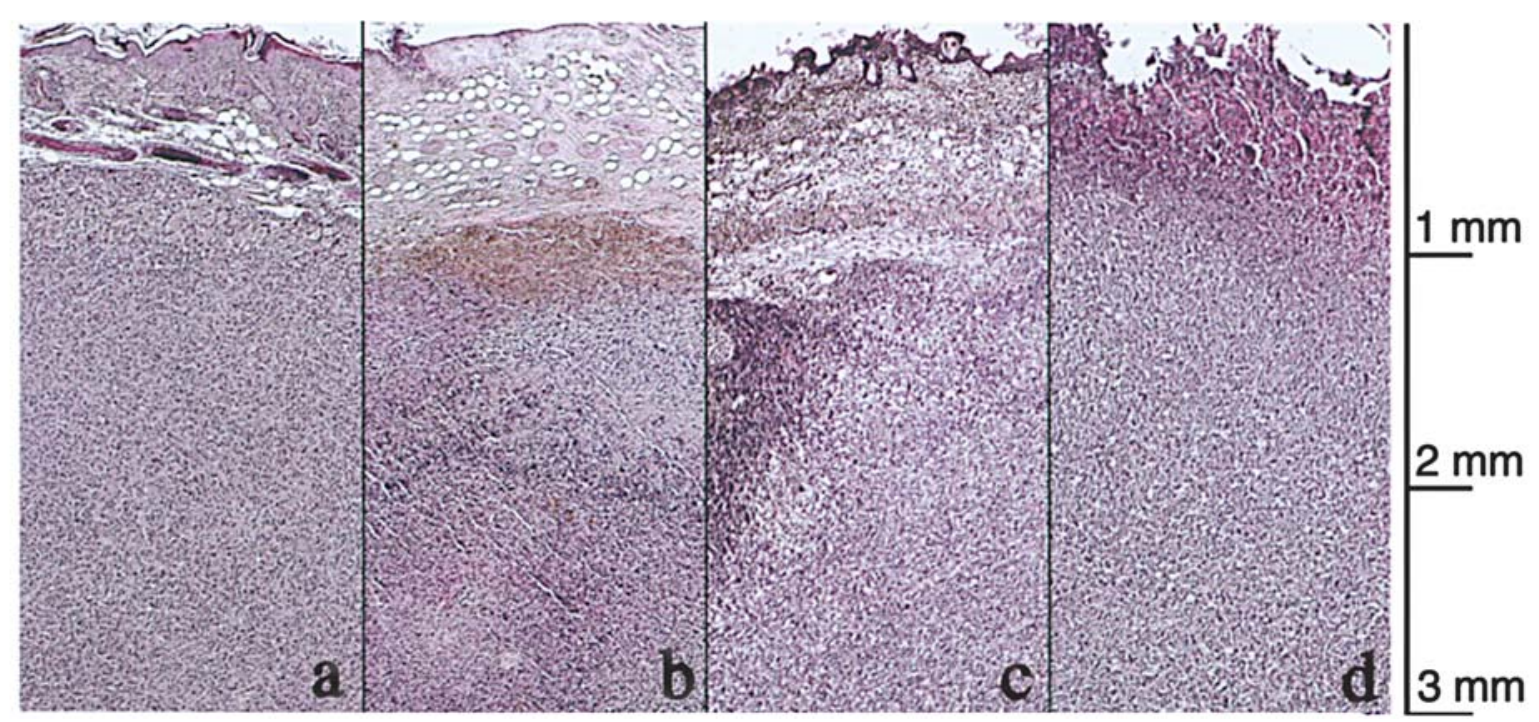

Figure 5. Histological findings. (a) Untreated control; (b) $24 \mathrm{~h}$ after HT-H (740 J/cm² irradiation and $40 \mathrm{~min}$ of heating); (c) $24 \mathrm{~h} \mathrm{after} \mathrm{PDT-H} \mathrm{(85} \mathrm{J/cm²}$ irradiation); (d) $24 \mathrm{~h}$ after PDT+HT-H (740 J/ $\mathrm{cm}^{2}$ irradiation and $40 \mathrm{~min}$ of heating). Original magnification, x60; hematoxylin-eosin staining.

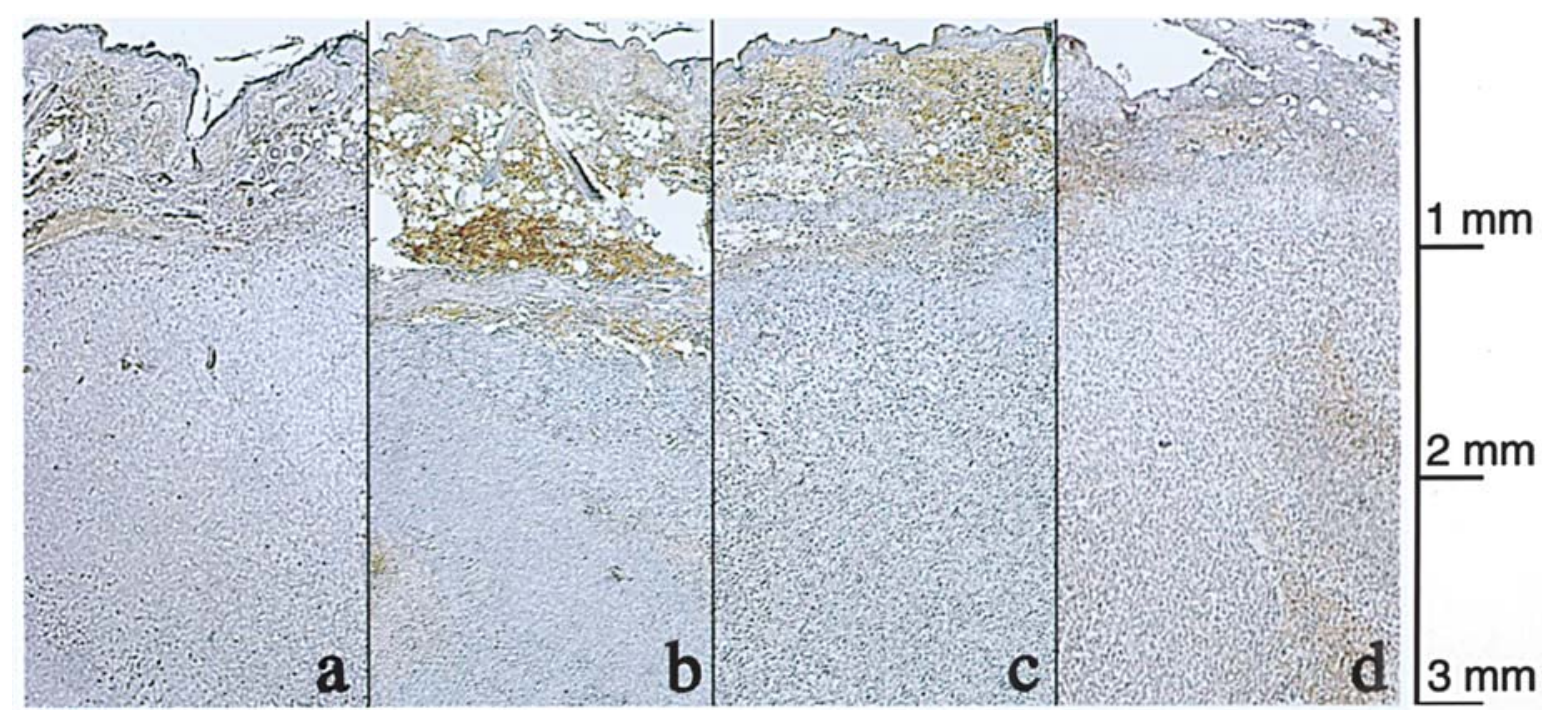

Figure 6. Immunohistochemical detection of Carbonic anhydrase IX expression. (a) untreated control; (b) $24 \mathrm{~h}$ after HT-H (740 J/cm² irradiation and $40 \mathrm{~min}$

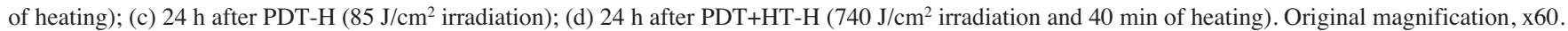

development of the next generation photosensitizers has been achieved, providing wider options for the clinical use of photosensitizers $(12,16,18-22)$.

Photofrin has been most widely used as a photosensitizer since its development in the 1990s. Since it is not particularly tumor specific and its washout time is relatively long, light shielding is needed for several weeks in clinical practice to avoid photosensitivity (16). On the other hand, 5-aminolevulinic acid (ALA), which has recently been used in clinical practice, is advantageous in that photosensitivity can be avoided, due to its excretion within $24 \mathrm{~h}$, and that repeated administration is possible because of its short washout time. Therefore, in PDT with ALA (ALA-PDT) there are many advantages in that the same lesion can be treated repeatedly with no effect on subsequent treatment and the QOL is not impaired, due to decreased burden on the patient, such as post-treatment damage of the skin $(3,11,12)$.

In addition to systemic oral and intravenous administration, local administration of ALA can be used without adverse effects, including vomiting and development of complications (e.g. depression of liver function and hypotension) that are observed upon systemic administration. Therefore, since 1990, when Kennedy et al used this therapy for skin disorders (23), ALA has been widely used for treatment and diagnosis of various superficial diseases, and many studies have been performed $(3,10,24-27)$. However, since ALA is administered locally as an application that has low permeability, the production of protoporphyrin IX (PPIX) is limited to the surface and ALA is considered to be ineffective for deepseated nodular tumors (10). In this study, local injection of 
ALA was selected as the administration route to avoid various complications as a result of systemic administration and to enable ALA to penetrate into deep-seated tumors. If ALAPDT, which has many advantages upon local administration, is effective for both superficial diseases and deep-seated diseases, broader treatment options will be available for neoplastic lesions, including malignant tumors. However, there are few in vivo studies of the therapy for deep-seated solid tumors $(13,14)$, and no studies of human-derived osteosarcoma have been reported.

On the other hand, hyperthermia (HT) alone is not an appropriate therapy for malignant tumors. Furthermore, the effectiveness of HT combined with other therapies, especially radiotherapy, has been confirmed. Several studies have been performed with combination therapy of HT and PDT for malignant tumors and non-neoplastic diseases, suggesting the effectiveness of HT in combination therapy $(11-14,17,28,29)$.

In this study, based on the results of previous studies (13), the effectiveness of combination therapy of ALA-PDT and HT on human-derived osteosarcoma was examined using a linearly polarized near infrared hyperthermic irradiator, Super Lizer (SL).

To examine the effectiveness of the combination therapy of PDT and HT with photosensitizers other than ALA, PDT using a hematoporphyrin derivative (Hpd) and HT with Nd:YAG Laser were performed on mammary tumors. Simultaneous use of these therapies demonstrated greater effectiveness than therapies in which HT was performed prior to PDT, or in which PDT was performed prior to HT, suggesting a synergistic interaction. However, the mechanism of this synergistic interaction remains unclear (28). Furthermore, when HT was performed immediately after PDT with hypericin, a synergistic interaction was confirmed. It has been reported that both PDT and HT can damage blood vessels, which is considered a secondary effect, and that increase in direct damage to tumor cells might contribute to the synergistic interaction (29).

On the other hand, in the examination of combination therapy of ALA-PDT and HT, intraperitoneal administration of ALA with near infrared HT was performed on mousederived colon carcinomas. The rates of inhibition of tumor growth on day 12 after the treatment were $67 \%$ in HT, 56\% in ALA-PDT, and $17 \%$ in PDT with HT, demonstrating synergistic interactions. It was considered that through the synergistic interaction, the effect of ALA-PDT was increased by mild HT that increased blood flow and caused oxidation of the tumors (13). Furthermore, in the examination on DSsarcoma, a synergistic interaction of combination therapy of ALA-PDT was confirmed using intraperitoneal administration of ALA and HT with near infrared ray. At the cellular level, the mechanism of the synergistic interaction may be through PDT enhancement of the cytotoxic effect of HT by decreasing glutathione and increasing the thermosensitivity of cells, while inhibiting the increase in expression of heat shock protein 70 and the heat defense system during HT (14).

As shown in previous in vitro studies, this study also demonstrated that both ALA-PDT and the combination of ALA-PDT and HT exhibited significant inhibitory effects on tumor growth. In the $\mathrm{H}$ group, with high total energy, the tumor growth inhibition rates (GIR) were $52.3 \%$ in PDT-H, and $27.3 \%$ in PDT+HT-H, demonstrating that the inhibitory effect on tumor growth in ALA-PDT was significantly increased by simultaneous use of HT. In addition, an effect equivalent to that in the examination on mouse-derived colon carcinoma (13) was confirmed, although there was a difference in total energy.

However, incomplete resolution of the tumor was confirmed in this study. To ensure greater effectiveness, an increase in the drug concentration and total energy of irradiation may be necessary. However, since this may cause adverse effects and require a longer irradiation period, the possibility of patients experiencing pain in clinical use should be considered. In PDT + HT-H, tumor growth was not demonstrated by day 9 of the treatment. As described above, since ALA can be administered repeatedly, further effectiveness may be ensured by repeatedly performing ALA-PDT simultaneously with HT at an interval of 8-10 days. In addition to examination of the inhibitory effects towards tumors by repeated application, tolerance, which is generally considered not to be observed in PDT, should also be investigated (30).

In general, the ineffectiveness of ALA-PDT on deep-seated tumors has been attributed to the local concentration of the photosensitizers and the penetration of radiation with sufficient energy to the target area (6). Oxygen supply to tumor cells during irradiation may also be an important factor, since PDT exhibits a direct anti-tumor effect on tumor cells through the production of active oxygen (3). In an environment with normal temperatures, blood flow in tumors is greater than that in the periphery of normal tissues. However, in the central part of the tumor, hypoxia can be observed, because blood flow is lowered due to insufficient development of blood vessels in the rapidly growing tumor cells (31). Therefore, it is considered difficult to ensure the effectiveness of PDT in the deep area of a tumor due to local hypoxia. It has been reported that combination therapy with hyperbaric oxygen was effective to increase oxygen concentrations in the deep tissues of tumors and may enhance the effect of PDT $(32,33)$. Furthermore, it has been estimated that increased blood flow of tumor vessels as a result of treatment may increase the oxygen supply and effectiveness of PDT. However, no studies on its effectiveness have been reported.

On the other hand, heating to $42^{\circ} \mathrm{C}$ for $30-40 \mathrm{~min}$ is considered to cause direct cell damage; although the response to HT varies depending on the type of tumor cell (8). In animal studies examining the effect on tumor vessels in rats and mice, it was reported that $\mathrm{HT}$ at $42.5^{\circ} \mathrm{C}$ or higher for $\sim 1 \mathrm{~h}$ caused a decrease in blood flow of tumor vessels and induced hypoxia, decreased $\mathrm{pH}$ and malnutrition in the tumor environment; although the level of such phenomena varied depending on the type of tumor (9). By contrast, in mild HT at lower temperatures, $38-41^{\circ} \mathrm{C}$, there were some cases in which blood flow initially increased by 1.5 -fold but decreased over time, resulting in the occlusion of blood vessels $(9,34)$.

In the histological findings of this study, slight necrosis was observed in the area from the surface to a depth of $2 \mathrm{~mm}$ after HT, but no significant changes were observed in the deeper layers, demonstrating that the inhibitory effect of HT on tumor growth was slight. In previous in vitro studies, heating at $43.5^{\circ} \mathrm{C}$ for $30-40$ min caused no lethal damage to osteosarcoma cells; a result that was also confirmed in this study (11). After ALA-PDT, necrosis was observed in the area from the surface to a depth of $2 \mathrm{~mm}$, and only a slight effect was confirmed in 
deeper layers. On the other hand, after PDT with simultaneous HT, necrosis was observed in layers even deeper than $2 \mathrm{~mm}$ below the surface.

Based on these findings, it is considered that, in the area from the surface to the depth of $2 \mathrm{~mm}$ in tumor tissues treated with ALA-PDT with simultaneous HT, ALA-PDT directly damaged the tumor cells, and HT exhibited a synergistic effect to damage tumor vessels, since the temperature of this area increased to $43^{\circ} \mathrm{C}$ or higher, with the net result that necrosis occurred in this area. In the layers deeper than $2 \mathrm{~mm}$, ALAPDT was effective because blood flow increased, due to the increase in temperature from 37 to $43^{\circ} \mathrm{C}$, and oxygen supply to the peripheral part of the tumor was increased. It can be estimated that the tumor necrosis was caused by a synergistic interaction of the effectiveness of ALA-PDT and that of HT that induced decreased blood flow or occlusion of the vessels by secondary damage of tumor vessels.

Carbonic anhydrase IX (CAIX) is a marker of hypoxia that increasingly develops in a hypoxic environment as a result of low $\mathrm{pH}(35,36)$. In this study, while weak expression of CAIX was observed after ALA-PDT in an area relatively close to the surface, a positive area extended to the deeper layers after PDT with simultaneous HT compared with ALA-PDT. The expression of CAIX mRNA begins to increase within $3 \mathrm{~h}$ of the development of hypoxia, and reaches a peak in 6-24 h (37). By contrast, cell death occurs 1-6 h after ALA-PDT. These findings demonstrate that expression of CAIX is not observed in the necrotic region following PDT (38). Based on these findings, it can be considered that, after combination therapy of PDT with HT, the necrosis in the deep area was caused as a result of hypoxia induced by the synergistic interaction of HT that decreased blood flow or occluded the vessels by damaging tumor vessels; although ALA-PDT caused direct cell damage that was not sufficiently lethal to cause instant cell death. To accurately confirm these findings, however, it will be necessary to examine changes in blood flow at a given depth in the tumor during and after the treatment, and or changes in the oxygen partial pressure. Furthermore, although simultaneous treatment was performed in this study, the effect of the treatment should be compared between cases in which HT is performed prior to PDT, and vice versa.

In PDT using Photofrin, the antitumor effect can be caused by the significant decrease in blood flow as a result of stenosis or occlusion of tumor vessels due to the damage to vascular endothelial cells, in addition to direct damage of the tumor cells. By contrast, in ALA-PDT, damage to tumor vessels that could induce a decrease in blood flow does not occur; although mild damage to vascular endothelial cells can be observed $(39,40)$. It is considered that ALA-PDT exhibits less effects than PDT using Photofrin since ALA-PDT therapy exhibits less effects on tumor vessels and blood flow (39). This decreased effect on blood vessels may become a favorable factor to increase blood flow by HT when ALA-PDT is performed concomitantly with HT. However, it has been reported that the antitumor effect is increased by synergistic interaction of combination therapy of PDT and HT, because the effect of these therapies to damage blood vessels induces lethal damage to tumor vessels (4). Therefore, to clarify the mechanism(s) of the synergistic interaction in PDT with HT it will be necessary to monitor and compare the damage on blood vessels in PDT performed not only with ALA but with Photofrin or other photosensitizers.

In conclusion, ALA-PDT with simultaneous HT, using SL for the irradiation demonstrated a significant inhibitory effect on tumor growth of human-derived osteosarcoma, and a synergistic interaction of simultaneous HT. This suggests the possibility that ALA-PDT is useful, not only for the treatment of superficial tumors, but also for treatment of deep-seated mesenchymal tumors, such as osteosarcoma. However, since osteosarcomas originate in bones, careful consideration is necessary in clinical practice. Furthermore, the mechanism of the synergistic interaction suggested that ALA-PDT was effective in the deep area of tumors due to the increase of blood flow by mild hyperthermia. However, since incomplete tumor disappearance was observed in this study, the depth at which the therapy can be effective should be further examined clinically. To ensure further effectiveness, repeated treatment is desirable for the QOL of patients rather than increasing the total energy. For this purpose, further investigation should be performed to examine the optimal conditions regarding treatment interval and frequency, taking into consideration the burden on patients during and after the treatment.

\section{References}

1. Brancaleon L and Moseley H: Laser and non-laser light sources for photodynamic therapy. Lasers Med Sci 17: 173-186, 2002.

2. Morgan J and Oseroff AR: Mitochondria-based photodynamic anti-cancer therapy. Adv Drug Deliv Rev 49: 71-86, 2001.

3. Peng Q, Warloe T, Berg K, Moan J, Kongshaug M, Giercksky KE and Nesland JM: 5-Aminolevulinic acid-based photodynamic therapy. Clinical research and future challenges. Cancer 79: 2282-2308, 1997

4. Liu DL, Andersson-Engels S, Sturesson C, Svanberg K, Hakansson $\mathrm{CH}$ and Svanberg S: Tumour vessel damage resulting from laser-induced hyperthermia alone and in combination with photodynamic therapy. Cancer Lett 111: 157-165, 1997.

5. Harmon BV, Corder AM, Collins RJ, Gobe GC, Allen J, Allan DJ and Kerr JF: Cell death induced in a murine mastocytoma by 42-47 degrees $\mathrm{C}$ heating in vitro: evidence that the form of death changes from apoptosis to necrosis above a critical heat load. Int J Radiat Biol 58: 845-858, 1990.

6. Remani P, Ostapenko VV, Akagi K, Bhattathiri VN, Nair MK and Tanaka Y: Relation of transmembrane potential to cell survival following hyperthermia in HeLa cells. Cancer Lett 144: 117-123, 1999.

7. Yuen WF, Fung KP, Lee CY, Choy YM, Kong SK, Ko S and Kwok TT: Hyperthermia and tumour necrosis factor-alpha induced apoptosis via mitochondrial damage. Life Sci 67: 725-732, 2000.

8. Dewey WC, Hopwood LE, Sapareto SA and Gerweck LE: Cellular responses to combinations of hyperthermia and radiation. Radiology 123: 463-474, 1977.

9. Song CW: Effect of local hyperthermia on blood flow and microenvironment: a review. Cancer Res 44: 4721-4730, 1984.

10. De Blois AW, Thissen MR, De Bruijn HS, Grouls RJ, Dutrieux RP, Robinson DJ and Neumann HA: In vivo pharmacokinetics of protoporphyrin IX accumulation following intracutaneous injection of 5-aminolevulinic acid. J Photochem Photobiol B 61: 21-29, 2001.

11. Yanase S, Nomura J, Matsumura Y, et al: Enhancement of the effect of 5-aminolevulinic acid-based photodynamic therapy by simultaneous hyperthermia. Int J Oncol 27: 193-201, 2005.

12. Nomura J, Yanase S, Matsumura Y, Nagai K and Tagawa T: Efficacy of combined photodynamic and hyperthermic therapy with a new light source in an in vivo osteosarcoma tumor model. J Clin Laser Med Surg 22: 3-8, 2004.

13. Orenstein A, Kostenich G, Kopolovic Y, Babushkina T and Malik Z: Enhancement of ALA-PDT damage by IR-induced hyperthermia on a colon carcinoma model. Photochem Photobiol 69: 703-707, 1999. 
14. Frank J, Lambert C, Biesalski HK, Thews O, Vaupel P and Kelleher DK: Intensified oxidative and nitrosative stress following combined ALA-based photodynamic therapy and local hyperthermia in rat tumors. Int J Cancer 20: 941-948, 2003.

15. Valeriote $\mathrm{F}$ and Lin $\mathrm{H}$ : Synergistic interaction of anticancer agents: a cellular perspective. Cancer Chemother Rep 59: 895-900, 1975.

16. Ma G, Ikeda H, Inokuchi T and Sano K: Effect of photodynamic therapy using 5-aminolevulinic acid on 4-nitroquinoline-1oxide-induced premalignant and malignant lesions of mouse tongue. Oral Oncol 35: 120-124, 1999.

17. Kelleher DK, Bastian J, Thews O and Vaupel P: Enhanced effects of aminolaevulinic acid-based photodynamic therapy through local hyperthermia in rat tumours. Br J Cancer 89: 405-411, 2003.

18. Young SW, Woodburn KW, Wright M, et al: Lutetium texaphyrin (PCI-0123): a near-infrared, water-soluble photosensitizer. Photochem Photobiol 63: 892-897, 1996.

19. Krebs I, Binder S, Stolba U, Glittenberg C, Brannath W and Goll A: Choroidal neovascularization in pathologic myopia: three-year results after photodynamic therapy. Am J Ophthalmol 140: 416-425, 2005.

20. Takahashi H, Itoh Y, Nakajima S, Sakata I and Iizuka H: A novel ATX-S10(Na) photodynamic therapy for human skin tumors and benign hyperproliferative skin. Photodermatol Photoimmunol Photomed 20: 257-265, 2005.

21. Lilge L and Wilson BC: Photodynamic therapy of intracranial tissues: a preclinical comparative study of four different photosensitizers. J Clin Laser Med Surg 16: 81-91, 1998.

22. Wong TW, Aizawa K, Sheyhedin I, Wushur C and Kato H: Pilot study of topical delivery of mono-L-aspartyl chlorin e6 (NPe6): implication of topical NPe6-photodynamic therapy. J Pharmacol Sci 93: 136-142, 2003.

23. Kennedy JC, Pottier RH and Pross DC: Photodynamic therapy with endogenous protoporphyrin IX: basic principles and present clinical experience. J Photochem Photobiol B 6: 143-148, 1990.

24. Dougherty TJ, Gomer CJ, Henderson BW, et al: Photodynamic therapy. J Natl Cancer Inst 90: 889-905, 1998.

25. Itoh Y, Ninomiya Y, Henta T, Tajima S and Ishibashi A: Topical delta-aminolevulinic acid-based photodynamic therapy for Japanese actinic keratoses. J Dermatol 27: 513-518, 2000.

26. Itoh Y, Henta T, Ninomiya Y, Tajima S and Ishibashi A: Repeated 5-aminolevulinic acid-based photodynamic therapy following electro-curettage for pigmented basal cell carcinoma. J Dermatol 27: 10-15, 2000.

27. Fink-Puches R, Wolf $\mathrm{P}$ and Kerl H: Photodynamic therapy of superficial basal cell carcinoma by instillation of aminolevulinic acid and irradiation with visible light. Arch Dermatol 133: 1494-1495, 1997.
28. Mang TS: Combination studies of hyperthermia induced by the neodymium: yttrium-aluminum-garnet (Nd:YAG) laser as an adjuvant to photodynamic therapy. Lasers Surg Med 10: 173-178, 1990.

29. Chen B, Roskams T and De Witte PA: Enhancing the antitumoral effect of hypericin-mediated photodynamic therapy by hyperthermia. Lasers Surg Med 3: 158-163, 2002.

30. Shackley DC, Haylett A, Whitehurst C, Betts CD, O'Flynn K, Clarke NW and Moore JV: Comparison of the cellular molecular stress responses after treatments used in bladder cancer. BJU Int 90: 924-932, 2002.

31. Wartenberg M, Donmez F, Ling FC, Acker H, Hescheler J and Sauer H: Tumor-induced angiogenesis studied in confrontation cultures of multicellular tumor spheroids and embryoid bodies grown from pluripotent embryonic stem cells. FASEB J 15: 995-1005, 2001

32. Al-Waili NS, Butler GJ, Beale J, Hamilton RW, Lee BY and Lucas P: Hyperbaric oxygen and malignancies: a potential role in radiotherapy, chemotherapy, tumor surgery and phototherapy. Med Sci Monit 11: 279-289, 2005.

33. Huang Z, Chen Q, Shakil A, Chen H, Beckers J, Shapiro H and Hetzel FW: Hyperoxygenation enhances the tumor cell killing of photofrin-mediated photodynamic therapy. Photochem Photobiol 78: 496-502, 2003.

34. Kelleher DK, Thews O, Scherz A, Salomon Y and Vaupel P: Combined hyperthermia and chlorophyll-based photodynamic therapy: tumour growth and metabolic microenvironment. Br J Cancer 89: 2333-2339, 2003.

35. Bussink J, Kaanders JH and van der Kogel AJ: Tumor hypoxia at the micro-regional level: clinical relevance and predictive value of exogenous and endogenous hypoxic cell markers. Radiother Oncol 67: 3-15, 2003.

36. Wykoff CC, Beasley NJ, Watson PH, et al: Hypoxia-inducible expression of tumor-associated carbonic anhydrases. Cancer Res 60: 7075-7083, 2000.

37. Sorensen BS, Hao J, Overgaard J, Vorum H, Honore B, Alsner J and Horsman MR: Influence of oxygen concentration and $\mathrm{pH}$ on expression of hypoxia induced genes. Radiother Oncol 76: 187-193, 2005.

38. Loh CS, Vernon D, MacRobert AJ, Bedwell J, Bown SG and Brown SB: Endogenous porphyrin distribution induced by 5aminolaevulinic acid in the tissue layers of the gastrointestinal tract. J Photochem Photobiol B 20: 47-54, 1993.

39. Herman MA, Fromm D and Kessel D: Tumor blood-flow changes following protoporphyrin IX-based photodynamic therapy in mice and humans. J Photochem Photobiol B 52: 99-104, 1999.

40. Chang CJ, Sun CH, Liaw LH, Berns MW and Nelson JS: In vitro and in vivo photosensitizing capabilities of 5-ALA versus photofrin in vascular endothelial cells. Lasers Surg 24: 178-186, 1999. 